\title{
Apparent treatment-resistant hypertension among ambulatory hypertensive patients: a cross-sectional study from 13 general hospitals
}

\author{
Sehun Kim ${ }^{1,{ }^{*} \dagger}$, Jin Joo Park ${ }^{2,}$, Mi-Seung Shin 3 , Choong Hwan Kwak ${ }^{4}$, Bong-Ryeol Lee ${ }^{5}$, Sung-Ji Park ${ }^{6}$, \\ Hae-Young Lee ${ }^{7}$, Sang-Hyun $\mathrm{Kim}^{8}$, Seok-Min Kang ${ }^{9}$, Byung-Su Yoo ${ }^{10}$, Joong-Wha Chung ${ }^{11}$, Si Wan $\mathrm{Choi}^{12}$, \\ Sang-Ho Jo ${ }^{13}$, Jinho Shin ${ }^{14}$, and Dong-Ju Choi ${ }^{2}$
}

\begin{abstract}
${ }^{1}$ Division of Cardiology, Department of Internal Medicine, Hallym University Hangang Sacred Heart Hospital, Seoul; ${ }^{2}$ Cardiovascular Center, Seoul National University Bundang Hospital, Seongnam; ${ }^{3}$ Department of Cardiology, Gachon University Gil Medical Center, Incheon; ${ }^{4}$ Division of Cardiology, Department of Internal Medicine, Gyeongsang National University Changwon Hospital, Changwon; ${ }^{5}$ Division of Cardiology, Department of Internal Medicine, Daegu Fatima Hospital, Daegu; ${ }^{6}$ Division of Cardiology, Department of Medicine, Samsung Medical Center, Sungkyunkwan University School of Medicine, Seoul; ${ }^{7}$ Division of Cardiology, Department of Internal Medicine, Seoul National University Hospital, Seoul; ${ }^{8}$ Division of Cardiology, Department of Internal Medicine, Seoul Metropolitan Government Seoul National University Boramae Medical Center, Seoul; ${ }^{9}$ Division of Cardiology, Department of Internal Medicine, Severance Hospital, Yonsei University College of Medicine, Seoul; ${ }^{10}$ Division of Cardiology, Department of Internal Medicine, Wonju Severance Christian Hospital, Yonsei University Wonju College of Medicine, Wonju; ${ }^{11}$ Division of Cardiology, Department of Internal Medicine, Chosun University Hospital, Gwangju; ${ }^{12}$ Division of Cardiology, Department of Internal Medicine, Chungnam National University Hospital, Daejeon; ${ }^{13}$ Division of Cardiology, Department of Internal Medicine, Hallym University Sacred Heart Hospital, Anyang; ${ }^{14}$ Division of Cardiology, Department of Internal Medicine, Hanyang University Hospital, Seoul, Korea
\end{abstract}

Background/Aims: To examine the prevalence and clinical characteristics of apparent treatment-resistant hypertension among ambulatory hypertensive patients.

Methods: We enrolled adult ambulatory hypertensive patients at 13 well-qualified general hospitals in Korea from January to June 2012. Apparent resistant hypertension was defined as an elevated blood pressure $>140 / 90 \mathrm{mmHg}$ with the use of three antihypertensive agents, including diuretics, or $\geq 4$ antihypertensives, regardless of the blood pressure. Controlled hypertension was defined as a blood pressure within the target using three antihypertensives, including diuretics.

Results: Among 16,915 hypertensive patients, 1,172 (6.9\%) had controlled hypertension, and 1,514 (8.9\%) had apparent treatment-resistant hypertension. Patients with apparent treatment-resistant hypertension had an earlier onset of hypertension (56.8 years vs. 58.8 years, $p=0.007)$ and higher body mass index $\left(26.3 \mathrm{~kg} / \mathrm{m}^{2}\right.$ vs. $24.9 \mathrm{~kg} / \mathrm{m}^{2}$, $p<0.001)$ than those with controlled hypertension. Drug compliance did not differ between groups. In the multivariable analysis, earlier onset of hypertension (odds ratio $[\mathrm{OR}], 0.98 ; 95 \%$ confidence interval [CI], 0.97 to $0.99 ; p<0.001$ ) and the presence of comorbidities (OR, 2.06; 95\% CI, 1.27 to 3.35; $p<0.001$ ), such as diabetes mellitus, ischemic heart disease, heart failure, and chronic kidney disease, were independent predictors. Among the patients with apparent treatment-resistant hypertension, only $5.2 \%$ were receiving $\geq 2$ antihypertensives at maximally tolerated doses.

Conclusions: Apparent treatment-resistant hypertension prevalence is $8.9 \%$ among ambulatory hypertensive patients in Korea. An earlier onset of hypertension and the presence of comorbidities are independent predictors. Optimization of medical treatment may reduce the rate of apparent treatment-resistant hypertension.

Keywords: Apparent treatment resistant hypertension; Prevalence; Characteristics; Independent predictor; Korea

Received: October 29, 2019, Revised: February 11, 2020, Accepted: February 17, 2020

\section{Correspondence to Dong-Ju Choi, M.D.}

Cardiovascular Center, Seoul National University Bundang Hospital, 82 Gumi-ro 173beongil, Bundang-gu, Seongnam 13620, Korea, Tel: +82-31-787-7007, Fax: +82-32-787-7041,

E-mail: djchoi@snubh.org, https://orcid.org/0000-0003-0146-2189

*These authors contributed equally to this work.

${ }^{\dagger}$ Current affiliation: Division of Cardiology, Seongnam Citizens Hospital, Seongnam, Korea 


\section{INTRODUCTION}

High blood pressure is associated with increased cardiovascular complications [1]. There is a large body of evidence showing that lowering blood pressure reduces cardiovascular events, and recently, even lower blood pressure targets were proposed [2,3]. However, more than half of the patients do not achieve the target blood pressure [4].

Among hypertensive patients, there exists a group of patients whose blood pressure is difficult to control. Failure to achieve blood pressure targets with $\geq 3$ antihypertensives (including diuretics) is defined as resistant hypertension [5]. Understanding the characteristics of patients with treatment-resistant hypertension is important, because they confer a patient group with an increased risk for cardiovascular events who may benefit from specialized care.

In order to diagnose true resistant hypertension, exact blood pressure measurements with 24-hour ambulatory blood pressure monitoring, confirmation of medication adherence, and exclusion of secondary hypertension are necessary [6]. Due to limitations of the outpatient clinic, apparent treatment-resistant hypertension (aTRH) is often used instead [7]. Patients with aTRH have increased cardiovascular mortality [8].

Nonetheless, until now, data on aTRH has been limited. The prevalence and clinical characteristics of aTRH are less known among ambulatory hypertensive patients in Korea, which we sought to elucidate in this study. In addition, we also planned to examine the adequacy of antihypertensive treatment with regards to prescribed drug combinations and doses, medication adherence, and physical activity.

\section{METHODS}

\section{Patients}

In this cross-sectional, multicenter study, adult hypertensive patients aged $\geq 20$ years at 13 well-qualified general hospitals in Korea were enrolled from January to June 2012 in an ambulatory care setting. This study was conducted in two phases. In the first phase, we retrospectively reviewed electronic medical records and screened for patients with aTRH and those with con- trolled hypertension $(\mathrm{CH})$. Then, we examined the prevalence of aTRH. In the second phase, we compared the clinical characteristics between patients with aTRH and those with $\mathrm{CH}$. Written informed consent was obtained from the participants in the second phase of the study.

Blood pressure was measured using a mercury sphygmomanometer (Baumanometer Desk model, W.A. Baum Co. Inc, Copiague, NY, USA) or Omron HBP-1300 (Omron Healthcare, Matsusaka, Japan) after the patients had rested for at least 5 minutes in a sitting position. We obtained two blood pressure measurements in both arms, at least 5 minutes apart, and assigned the higher value as the patient's blood pressure.

A systemic medical chart review was performed to categorize the patients according to the number of prescribed antihypertensives and blood pressure. We were especially interested in the patients whose blood pressure was difficult to control. Therefore, we only included patients who were taking $\geq 3$ antihypertensives. The dose of antihypertensives was converted into the equivalent dose of standard medications of each drug class, and the maximally tolerated dose was defined arbitrarily (twice the conventional dose of hypertension treatment) as follows: angiotensin-converting-enzyme inhibitor (ACEi): ramipril $10 \mathrm{mg}$; angiotensin receptor blocker (ARB): candesartan $32 \mathrm{mg}$; beta blocker (BB): carvedilol $50 \mathrm{mg}$; calcium channel blocker (CCB): amlodipine 10 $\mathrm{mg}$; and diuretics: hydrochlorothiazide $50 \mathrm{mg}$. The clinical characteristics and drug compliance were evaluated using a structured questionnaire and the Morisky medication adherence scale (MMAS-8-items). Those with < 6, 6-8, and 9-10 points were defined as low, moderate, and high compliance, respectively. Data on comorbidities (i.e., diabetes mellitus, dyslipidemia, ischemic heart disease, chronic renal and adrenal disease, and cerebrovascular disease), body mass index, and status of alcohol consumption, smoking, and physical activity were collected using an electronic case report form. The status of smoking was categorized as follows: current smoker, ex-smoker, and never smoker. Physical activity was also categorized into five groups depending on the frequency of their activity (never, once per month, once per week, two to four times per week, and daily). This study was approved by the Institutional Review Board (IRB) of each participating hospital (representative Institution: Seoul National University Bundang Hospital, IRB No. 
B-1210-176-302). The study complied with the Declaration of Helsinki.

\section{Definitions}

a'RH was defined as a blood pressure $>140 / 90 \mathrm{mmHg}$ (in cases of diabetes mellitus or chronic kidney disease, $>130 / 80 \mathrm{mmHg}$ ) with the use of three antihypertensives of three different classes, including a diuretic, or $\geq 4$ antihypertensives, regardless of the blood pressure according to the Seventh Report of the Joint National Committee (JNC-7) guidelines [9]. $\mathrm{CH}$ was defined as a blood pressure within the target with the use of three antihypertensives, including a diuretic.

The primary endpoint was the prevalence of aTRH. The secondary endpoint was the differences in the clinical characteristics between patients with aTRH and those with $\mathrm{CH}$.

\section{Statistical analysis}

Assuming that the prevalence of aTRH is as high as 10\% and that of $\mathrm{CH}$ is $3 \%, 1,380$ patients with aTRH and 420 patients with $\mathrm{CH}$ would be available for the assessment of patient characteristics when 13,800 hypertensive patients are screened. The sample size would allow a confidence interval of $<1 \%$ [10].

Data were presented as numbers and frequencies for categorical variables and as means \pm standard deviations for continuous variables. For comparison between groups, a chi-square test (or Fisher exact test when any expected count was $<5$ for a $2 \times 2$ table) for categorical variables and an unpaired Student $t$ test or one-way analysis of variance for continuous variables were applied. A two-sided $p$ value $<0.05$ was considered statistically significant. All analyses were performed using SAS version 9.2 (SAS Institute Inc., Cary, NC, USA) by a professional statistic council.

\section{RESULTS}

\section{Prevalence of apparent treatment resistant hyper- tension}

A total of 16,915 hypertensive patients were screened, and 2,686 patients were taking $\geq 3$ hypertensives, including a diuretic; 1,172 patients $(6.9 \%)$ had $\mathrm{CH}$, while 1,514 patients $(8.9 \%)$ had aTRH. Among the patients

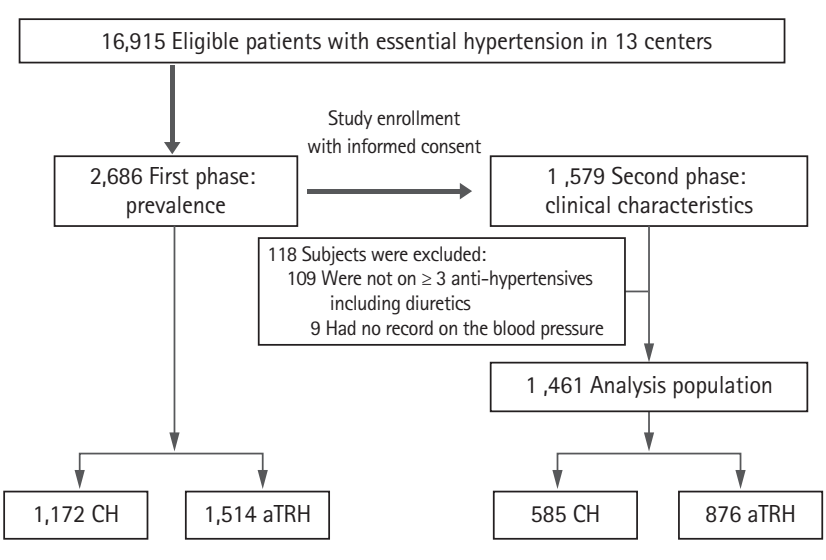

Figure 1. Study population. CH, controlled hypertension; aTRH, apparent treatment-resistant hypertension.

with aTRH, 542 patients (3.2\%) did not reach the blood pressure target with three antihypertensives, including diuretics, whereas 972 patients (5.7\%) were taking $\geq 4$ antihypertensives (Fig. 1).

Among the patients with aTRH, only 34\% achieved both systolic and diastolic blood pressure targets. Furthermore, $40.4 \%$ of the patients achieved the systolic blood pressure target, and 70.8\% achieved the diastolic blood pressure target.

\section{Clinical characteristics of the patients}

One thousand five hundred and seventy-nine patients filled out the self-questionnaire. Of them, 118 patients were excluded owing to violation of the inclusion criteria: nine patients received three antihypertensives but they did not have a blood pressure value and 109 did not meet inclusion criteria 2, which was using more than three different antihypertensives including diuretics. Therefore, the data of 1,461 patients were available for the analysis of patient clinical characteristics: 876 patients had aTRH, and 585 had $\mathrm{CH}$.

The patients with aTRH had an earlier onset of hypertension ( 56.8 years vs. 58.8 years, $p=0.007$ ) and longer hypertension duration $(9.2 \pm 7.2$ years vs. $8.3 \pm 6.6$ years, $p=$ 0.019). They also had a higher body mass index $(26.3 \mathrm{~kg} /$ $\mathrm{m}^{2}$ vs. $24.9 \mathrm{~kg} / \mathrm{m}^{2}, \mathrm{p}<0.001$ ) than those with $\mathrm{CH}$ and had more comorbidities, such as diabetes mellitus (33.6\% vs. $26.2 \%, p=0.003)$, angina ( $28.3 \%$ vs. $22.2 \%, p<0.001)$, and chronic kidney disease ( $4.3 \%$ vs. $2.2 \%, p=0.031)$. With regards to lifestyle, alcohol consumption and cigarette smoking did not differ between groups. Interestingly, 
Table 1. Baseline clinical characteristics

\begin{tabular}{|c|c|c|c|c|}
\hline Variable & aTRH $(n=876)$ & $\mathrm{CH}(\mathrm{n}=585)$ & All $(n=1,461)$ & $p$ value \\
\hline Age, yr & $66.1 \pm 12.2$ & $67.1 \pm 11.4$ & $66.5 \pm 11.9$ & 0.145 \\
\hline Male sex, \% & 50.7 & 47.9 & 49.6 & 0.291 \\
\hline Body mass index, $\mathrm{kg} / \mathrm{m}^{2}$ & $26.3 \pm 3.9$ & $24.9 \pm 3.9$ & $25.8 \pm 3.9$ & $<0.001$ \\
\hline Age at diagnosis of $\mathrm{HT}, \mathrm{yr}$ & $56.8 \pm 13.0$ & $58.8 \pm 12.3$ & $57.6 \pm 12.7$ & 0.007 \\
\hline HT duration, yr & $9.2 \pm 7.2$ & $8.3 \pm 6.6$ & $8.9 \pm 7.0$ & 0.019 \\
\hline SBP, $\mathrm{mmHg}$ & $135.1 \pm 16.9$ & $118.9 \pm 11.4$ & $128.4 \pm 16.9$ & $<0.001$ \\
\hline $\mathrm{DBP}, \mathrm{mmHg}$ & $77 \pm 12.2$ & $70.0 \pm 9.2$ & $74.1 \pm 11.6$ & $<0.001$ \\
\hline Pulse pressure, $\mathrm{mmHg}$ & $58.0 \pm 14.1$ & $49.0 \pm 9.3$ & $54.3 \pm 13.1$ & $<0.001$ \\
\hline \multicolumn{5}{|l|}{ Goal BP achievement } \\
\hline SBP & $332(40.4)$ & $585(100)$ & $917(65.2)$ & $<0.001$ \\
\hline DBP & $581(70.8)$ & $585(100)$ & $1,166(82.9)$ & $<0.001$ \\
\hline SBP and DBP & $279(34)$ & $585(100)$ & $864(61.5)$ & $<0.001$ \\
\hline Pulse rate, beats/min & $73.6 \pm 13.2$ & $75 \cdot 7 \pm 13 \cdot 3$ & $74.5 \pm 13.2$ & 0.014 \\
\hline Comorbidity & $836(95.4)$ & $540(92.3)$ & $1,376(94.2)$ & 0.012 \\
\hline Diabetes & $294(33.6)$ & $153(26.2)$ & $447(30.6)$ & 0.003 \\
\hline Dyslipidemia & $479(54.7)$ & $291(49.7)$ & $770(52.7)$ & 0.064 \\
\hline Angina & $248(28.3)$ & $130(22.2)$ & $378(25.9)$ & $<0.001$ \\
\hline Heart failure & $219(25)$ & $118(20.2)$ & $337(23.1)$ & 0.031 \\
\hline Chronic kidney disease & $38(4 \cdot 3)$ & $13(2.2)$ & $51(3.5)$ & 0.031 \\
\hline Intracranial hemorrhage & $8(0.9)$ & $8(1.4)$ & $16(1.1)$ & 0.413 \\
\hline Stroke & $37(4.2)$ & $20(3.4)$ & $57(3.9)$ & 0.436 \\
\hline Respiratory disease & $52(5.9)$ & $40(6.8)$ & $92(6.3)$ & 0.487 \\
\hline Family history & & & & 0.934 \\
\hline $\mathrm{HT}$ & $168(19.2)$ & $108(18.5)$ & $276(18.9)$ & \\
\hline Diabetes & $57(6.5)$ & $38(6.5)$ & $95(6.5)$ & \\
\hline Dyslipidemia & $2(0.2)$ & $1(0.2)$ & $3(0.2)$ & \\
\hline Cardiovascular disease & $16(1.8)$ & $17(2.9)$ & $33(2.3)$ & \\
\hline Renal disease & $4(0.5)$ & $1(0.2)$ & $5(0.3)$ & \\
\hline Cerebrovascular disease & $25(2.9)$ & $22(3.8)$ & $47(3.2)$ & \\
\hline Respiratory disease & $11(1.3)$ & $7(1.2)$ & $18(1.2)$ & \\
\hline Alcohol consumption & $540(61.9)$ & $343(58.8)$ & $883(60.6)$ & 0.258 \\
\hline Smoking & & & & 0.321 \\
\hline Current & $100(11.5)$ & $53(9.1)$ & $153(10.5)$ & \\
\hline Ex-smoker & $258(29.6)$ & $183(31.4)$ & $441(30.3)$ & \\
\hline Never smoker & $514(58.9)$ & $347(59.5)$ & $861(59.2)$ & \\
\hline Vigorous physical exercise & & & & 0.005 \\
\hline Never & $622(76.5)$ & $462(79.2)$ & $1,084(77.7)$ & \\
\hline $1 / \mathrm{mo}$ & $65(8.0)$ & $23(3.9)$ & $88(6.3)$ & \\
\hline $1 /$ wk & $56(6.9)$ & $38(6.5)$ & $64(4.6)$ & \\
\hline $2-4 / w k$ & $72(8.9)$ & $38(6.5)$ & $110(7.9)$ & \\
\hline Daily & $52(6.4)$ & $22(3.8)$ & $74(5 \cdot 3)$ & \\
\hline Drug compliance (MMAS) & & & & 0.116 \\
\hline $\operatorname{Low}(<6)$ & $106(12.3)$ & $52(9.2)$ & $158(11.1)$ & \\
\hline Moderate ( 6 or 7 ) & $317(36.9)$ & $203(35.9)$ & $520(36.5)$ & \\
\hline $\operatorname{High}(8)$ & $436(50.8)$ & $311(54.9)$ & $747(52.4)$ & \\
\hline
\end{tabular}

Values are presented as mean \pm standard deviation or number (\%).

aTRH, apparent treatment-resistant hypertension; $\mathrm{CH}$, controlled hypertension; HT, hypertension; SBP, systolic blood pressure; DBP, diastolic blood pressure; BP, blood pressure; MMAS, Morisky medication adherence scale.

${ }^{a} t$ test or chi-square test was used. 
Table 2. Univariate and multivariate analysis for the predictors of apparent treatment-resistant hypertension

\begin{tabular}{|c|c|c|c|c|c|c|}
\hline \multirow{2}{*}{ Variable } & \multicolumn{3}{|c|}{ Univariate } & \multicolumn{3}{|c|}{ Multivariate $^{\mathrm{a}}$} \\
\hline & Odds ratio & $95 \% \mathrm{CI}$ & $p$ value & Odds ratio & $95 \% \mathrm{CI}$ & $p$ value \\
\hline Age & 0.99 & $0.98-1.00$ & 0.145 & & & \\
\hline Male sex & 0.89 & $0.72-1.10$ & 0.290 & & & \\
\hline Body mass index & 1.09 & $1.03-1.15$ & 0.001 & & & \\
\hline Age at diagnosis of $\mathrm{HT}$ & 0.98 & $0.97-0.99$ & 0.006 & 0.98 & $0.97-0.99$ & $<0.001$ \\
\hline HT duration & 1.02 & $1.00-1.03$ & 0.020 & & & \\
\hline Comorbidity & 1.74 & $1.12-2.70$ & 0.013 & 2.06 & $1.27-3 \cdot 35$ & $<0.001$ \\
\hline Diabetes & 1.42 & $1.13-1.79$ & 0.002 & 1.32 & $1.01-1.72$ & 0.037 \\
\hline Ischemic heart disease & 1.32 & $1.05-1.66$ & 0.015 & 1.30 & $1.00-1.69$ & 0.046 \\
\hline Heart failure & 1.31 & $1.02-1.70$ & 0.031 & 1.60 & $1.20-2.12$ & 0.001 \\
\hline Chronic kidney disease & 1.95 & $1.05-3.61$ & 0.031 & 2.93 & $1.34-6.41$ & 0.007 \\
\hline Concurrent medications & 1.08 & $0.79-1.47$ & 0.601 & & & \\
\hline Alcohol consume & 1.13 & $0.91-1.40$ & $0.25^{8}$ & & & \\
\hline \multicolumn{7}{|l|}{ Smoking } \\
\hline Current smoker & 1.27 & $0.88-1.82$ & 0.138 & & & \\
\hline Ex-smoker & 0.95 & $0.75-1.20$ & 0.201 & & & \\
\hline \multicolumn{7}{|l|}{ Physical activity ${ }^{\mathrm{b}}$} \\
\hline Light activity & 0.96 & $0.69-1.33$ & 0.071 & & & \\
\hline Moderate activity & 1.19 & $0.84-1.68$ & 0.377 & & & \\
\hline Heavy activity & 1.68 & $0.95-2.97$ & 0.652 & & & \\
\hline Extreme activity & $3 \cdot 59$ & $0.41-31.33$ & 0.311 & & & \\
\hline Family history & 0.99 & $0.77-1.26$ & 0.934 & & & \\
\hline \multicolumn{7}{|l|}{ Drug compliance (MMAS) ${ }^{c}$} \\
\hline$<6$ & 1.45 & $1.01-2.08$ & 0.073 & & & \\
\hline 6 or 7 & 1.11 & $0.88-1.40$ & 0.538 & & & \\
\hline
\end{tabular}

CI, confidence interval; HT, hypertension; MMAS, Morisky medication adherence scale.

${ }^{a}$ The multivariate model was adjusted for diabetes, hyperlipidemia, angina or myocardial infarction, congestive heart failure, atrial fibrillation, chronic kidney disease, cerebrovascular disease, and obstructive sleep apnea.

${ }^{\mathrm{b}}$ Physical activity was adjusted for resting as a reference value.

${ }^{\mathrm{c}}$ Drug compliance was adjusted for MMAS scores of 8-10 as reference values.

the patients with aTRH had more frequent physical activities than those with $\mathrm{CH}$. Drug compliance did not differ between groups (Table 1).

In the multivariable analysis, earlier onset of hypertension (odds ratio [OR], 0.98; 95\% confidence interval [CI], 0.97 to $0.99 ; p<0.001)$ and the presence of diabetes mellitus (OR, 1.32; 95\% CI, 1.01 to 1.72; $p=0.037$ ), ischemic heart disease (OR, 1.30; $95 \% \mathrm{CI}, 1.00$ to $1.69 ; p=0.046$ ), heart failure (OR, 1.60; $95 \% \mathrm{CI}, 1.20$ to $2.12 ; p=0.001)$, and chronic kidney disease (OR, 2.93; 95\% CI, 1.34 to $6.41 ; p=0.007$ ) were independent predictors of resistant hypertension (Table 2).

\section{Antihypertensive medications}

At the time of study enrollment, patients had 3-drug (43\%), 4-drug (39.5\%), and 5-drug (2.5\%) combinations. Among the patients with a 3 -drug combination, the most frequently prescribed combination was $\mathrm{ARB}+$ CCB (20.7\%), followed by ARB + BB (10.8\%), including diuretics in the aTRH group and ARB + CCB (47.2\%), followed by $\mathrm{ARB}+\mathrm{BB}(37.9 \%)$, including diuretics in the $\mathrm{CH}$ group (Table 3).

Among the patients with aTRH, 64.4\%, 29.3\%, 0.9\%, and $0.1 \%$ of the patients had none, one, two, and three 
Table 3. Antihypertensive drug combination in both groups

\begin{tabular}{|c|c|c|c|}
\hline Variable & $\operatorname{aTRH}(\mathrm{n}=876)$ & $\mathrm{CH}(\mathrm{n}=585)$ & $p$ value $^{\mathrm{a}}$ \\
\hline Three-drug combination including diuretics & $346(39.5)$ & $585(100)$ & $<0.001$ \\
\hline $\mathrm{ARB}+\mathrm{CCB}$ & $181(20.7)$ & $276(47.2)$ & $<0.001$ \\
\hline $\mathrm{ARB}+\mathrm{BB}$ & $95(10.8)$ & $223(37 \cdot 9)$ & $<0.001$ \\
\hline $\mathrm{ACEi}+\mathrm{BB}$ & $29(3 \cdot 3)$ & $38(6.5)$ & 0.004 \\
\hline $\mathrm{BB}+\mathrm{CCB}$ & $26(3.0)$ & $31(5 \cdot 3)$ & 0.024 \\
\hline $\mathrm{ACEi}+\mathrm{CCB}$ & $13(1.5)$ & $15(2.6)$ & 0.140 \\
\hline $\mathrm{ACEi}+\mathrm{ARB}$ & $2(0.2)$ & $1(0.2)$ & 0.812 \\
\hline $\mathrm{ARB}+\mathrm{AB}$ & 0 & $2(0.3)$ & 0.083 \\
\hline Four-drug combination including diuretics & $492(56.2)$ & & \\
\hline $\mathrm{ARB}+\mathrm{BB}+\mathrm{CCB}$ & $422(48.2)$ & & \\
\hline $\mathrm{ACEi}+\mathrm{BB}+\mathrm{CCB}$ & $37(4.2)$ & & \\
\hline $\mathrm{ACE} i+\mathrm{ARB}+\mathrm{CCB}$ & $20(2.3)$ & & \\
\hline $\mathrm{ACEi}+\mathrm{ARB}+\mathrm{BB}$ & $5(0.6)$ & & \\
\hline $\mathrm{ARB}+\mathrm{AB}+\mathrm{CCB}$ & $3(0.3)$ & & \\
\hline $\mathrm{ARB}+\mathrm{BB}+\mathrm{AB}$ & $2(0.2)$ & & \\
\hline $\mathrm{ACE}+\mathrm{BB}+\mathrm{AB}$ & $1(0.1)$ & & \\
\hline $\mathrm{ACEi}+\mathrm{CCB}+$ other & $1(0.1)$ & & \\
\hline $\mathrm{BB}+\mathrm{CCB}+$ other & $1(0.1)$ & & \\
\hline Five-drug combination including diuretics & $37(4.2)$ & & \\
\hline $\mathrm{ACEi}+\mathrm{ARB}+\mathrm{BB}+\mathrm{CCB}$ & $19(2.2)$ & & \\
\hline $\mathrm{ARB}+\mathrm{BB}+\mathrm{AB}+\mathrm{CCB}$ & $13(1.5)$ & & \\
\hline $\mathrm{ARB}+\mathrm{BB}+\mathrm{CCB}+$ other & $3(0.3)$ & & \\
\hline $\mathrm{ACEi}+\mathrm{ARB}+\mathrm{BB}+\mathrm{AB}$ & $1(0.1)$ & & \\
\hline $\mathrm{ACEi}+\mathrm{BB}+\mathrm{AB}+\mathrm{CCB}$ & $1(0.1)$ & & \\
\hline Seven-drug combination including diuretics & $1(0.1)$ & & \\
\hline $\mathrm{ACEi}+\mathrm{ARB}+\mathrm{BB}+\mathrm{AB}+\mathrm{CCB}+$ other & $1(0.1)$ & & \\
\hline
\end{tabular}

Values are presented as number (\%).

aTRH, apparent treatment-resistant hypertension; CH, controlled hypertension; ARB, angiotensin receptor blocker; CCB, calcium channel blocker; BB, beta blocker; ACEi, angiotensin-converting enzyme inhibitor; AB, alpha blocker.

${ }^{\mathrm{a} C h i-s q u a r e ~ t e s t ~ w a s ~ u s e d . ~}$

drugs at maximally tolerated doses, respectively. Of the drug classes, $53 \%$ of the CCBs were prescribed at maximally tolerated doses; in contrast, only $5.9 \%$ of the BBs were prescribed at maximally tolerated doses (Table 4).

\section{DISCUSSION}

In this study, we determined the prevalence of aTRH among ambulatory hypertensive patients at tertiary hospitals in Korea. The estimated prevalence of aTRH was $8.9 \%$, and the independent predictors of aTRH included early onset of hypertension and the presence of other comorbidities, including diabetes mellitus, ischemic heart disease, and chronic kidney disease. Among the patients with resistant hypertension, almost none of the patients received all antihypertensive drugs at maximally tolerated doses. Alcohol consumption, cigarette smoking, and drug compliance did not differ between patients with resistant and $\mathrm{CH}$. 
Table 4. Antihypertensive medications at maximally tolerated dose

\begin{tabular}{lcc}
\hline Variable & $\begin{array}{c}\mathrm{aTRH}^{\mathrm{a}} \\
(\mathrm{n}=864)\end{array}$ & $\begin{array}{c}\mathrm{CH}^{\mathrm{a}} \\
(\mathrm{n}=575)\end{array}$ \\
\hline No. of drugs at MTD & & \\
0 & $556(64.4)$ & $439(76.3)$ \\
1 & $253(29.3)$ & $127(22.1)$ \\
2 & $46(0.9)$ & $9(1.6)$ \\
$>3$ & $9(0.1)$ & \\
Drug class at MTD, \% & & \\
ACEi & 17.4 & \\
ARB & 7.2 & \\
BB & 5.9 \\
CCB & 53.0 \\
Diuretic & 7.3 \\
\hline
\end{tabular}

aTRH, apparent treatment-resistant hypertension; $\mathrm{CH}$, controlled hypertension; MTD, maximally tolerated dose; ACEi, angiotensin-converting enzyme inhibitor; ARB, angiotensin receptor blocker; $\mathrm{BB}$, beta blocker; $\mathrm{CCB}$, calcium channel blocker.

${ }^{a}$ Twenty-two subjects whose medication doses were not available were excluded ( 12 subjects in the aTRH group, and 10 subjects in the $\mathrm{CH}$ group).

\section{Prevalence of apparent treatment-resistant hyper- tension}

Several types of studies provided estimates on the prevalence of resistant hypertension. In population-based studies with blood pressure control data, the prevalence ranged between $12 \%$ and $17 \%$ [11]. In outcome-based trials, it ranged between $8.4 \%$ and $14.9 \%$; in population-based studies, the prevalence ranged between $12.8 \%$ and $16.4 \%[6,12,13]$. Our study showed that the prevalence of aTRH was $8.9 \%$. Another study using the Korean population reported the prevalence of resistant hypertension as high as $7.9 \%$ in a primary care setting [14]. Choi et al. [15] conducted a prospective multicenter observational study in the Korean elderly population (> 60 years old) who underwent evaluation of secondary hypertension and reported the prevalence of aTRH as $19.1 \%$. In the most recent study using the Korean ambulatory blood pressure monitoring registry (Kor-ABP registry) data, Choi et al. [16] reported that the prevalence of resistant hypertension was $11.9 \%$. The differences may result from the different treatment settings (patients at primary versus tertiary centers, secondary hypertension clinic), study population, treatment duration, and the ambiguity of the definition of resistant hypertension.

\section{Characteristics of the study population}

Among the patients with aTRH, 40.4\% and 70.8\% achieved the systolic and diastolic blood pressure targets, respectively, implying that systolic blood pressure is the primary determinant of poor blood pressure control. Difficulty to control systolic blood pressure is also observed in other large hypertension trials and several factors, such as age, obesity, and target organ damage, are associated with an increased systolic blood pressure $[17,18]$. Because obesity is a modifiable risk factor, it is also an important treatment target [19,20].

An early onset of hypertension was associated with an increased risk for aTRH. Interestingly, the age of patients with and without aTRH did not differ, suggesting that patients with aTRH had a longer duration of hypertension. Other important predictors include the presence of comorbidities, such as diabetes mellitus and chronic kidney disease, which was consistent with those of previous studies from the National Health and Nutrition Examination Survey 1988 to 2008 [6,21]. The development of resistant hypertension is multifactorial and constitutional, and genetic factors play an important role. With an aging society and increasing prevalence of diabetes mellitus and chronic kidney disease, the prevalence of resistant hypertension is expected to increase as well [21].

\section{Drug combination and dose}

Forced up-titration of antihypertensives of three different classes, including a diuretic, is important for the diagnosis of resistant hypertension. One important finding of this study is that physicians do not prescribe maximally tolerated doses, indicating that these highrisk patients remain undertreated. Among the different drug classes, CCB was the most frequently prescribed (53\%), and BB was the least frequently (5.9\%) prescribed at maximally tolerated doses, suggesting that physicians are especially comfortable with a dose escalation of CCBs.

Fifty-six percent of patients with resistant hypertension had a 4-drug combination, and the most common combination was ARB or ACEi + BB + CCB + diuretics. In the PATHWAY-2 trial, the use of spironolactone as 
the fourth antihypertensive agent was the most effective add-on drug to the treatment with ACEI or ARB + CCB + thiazide-like diuretic [22]. In this study, only $10.6 \%$ received spironolactone. Considering that only few modifiable risk factors exist, careful drug combination and appropriate up-titration would improve blood pressure control [12,19,23].

\section{Drug compliance and lifestyle modification}

Interestingly, drug compliance, obesity, alcohol consumption, smoking, and physical activity were not independent risk factors of resistant hypertension. Drug non-adherence is a major reason for failed blood pressure control, and up to $40 \%$ of patients discontinued their antihypertensives in the first year of treatment [2426]. Drug compliance was high in both groups in this study, which is also often observed at tertiary hospitals [27]. Obesity is a well-known risk factor for hypertension, and its degree is directly related to the degree of blood pressure [23]. The relatively low body mass index in this study population compared with those from Western studies may be the reason for the lack of association. In previous studies, only heavy drinkers had an increased risk for hypertension; however, in this study, the proportion of heavy drinkers was low and did not differ between groups, thus explaining the neutral association.

With regards to physical activity, light and moderate aerobic exercises can reduce ambulatory blood pressure $[28,29]$. The majority of patients in this study participated in light daily exercises in both groups.

\section{Study limitations}

Our study has several limitations. First, to avoid pseudo-resistant hypertension owing to poor adherence or white coat hypertension, reliable blood pressure measurements and drug adherence are very crucial. In this study, we did not perform 24-hour ambulatory blood pressure monitoring or workups to exclude secondary hypertension. Thus, our definition conforms to the definition of aTRH. Second, many patients had angina or heart failure, and may have received BBs, RAS blockers or diuretics to treat the underlying disease and may have been falsely classified as having resistant hypertension. In the Kor-ABP registry, coronary artery disease and heart failure were consistently associated with $\mathrm{CH}$ which is attributed to the routine use of BBs, RAS blockers, and diuretics [16]. In our study the proportion of patients with angina and heart failure was 53.3\% in the aTRH group and $42.4 \%$ in the $\mathrm{CH}$ group which mandates careful interpretation of the study findings. Third, because the study was conducted at 13 qualified general hospitals (including a teaching institutional hospital), selection bias may exist and the prevalence of aTRH may be underestimated [6]. Nonetheless, aTRH itself is a predictor of worse clinical outcomes and understanding the characteristics of patients with aTRH is clinically important. It is of note that the prevalence of true resistant hypertension may be lower in our study population [8].

Although we did not perform a "pill count," the drug adherence was measured using the structured questionnaire with the MMAS, a surrogate with limitations. At last, because we only enrolled ambulatory hypertensive patients at tertiary centers in Asia, we do not know whether the study findings can be directly correlated to other populations with different ethnicities and those from other regions. Finally, because the definition of resistant hypertension has been modified in the newly updated clinical practice guideline, further studies are necessary to address the recent updates [30,31].

In conclusions, the prevalence of aTRH is $8.9 \%$ among ambulatory hypertensive patients in Korea. Early onset and the presence of comorbidities are independent predictors. Many patients with aTRH do not receive adequate hypertension management. An appropriate up-titration with an optimal combination of antihypertensives may reduce the prevalence of resistant hypertension.

\section{KEY MESSAGE}

1. The prevalence of apparent treatment-resistant hypertension is $8.9 \%$ among ambulatory hypertensive patients in Korea.

2. Many patients with apparent treatment-resistant hypertension do not receive adequate hypertension management.

3. An appropriate up-titration with an optimal combination of antihypertensives may reduce the prevalence of resistant hypertension. 


\section{Conflict of interest}

This research was funded by Handok Inc.

\section{REFERENCES}

1. Lewington S, Clarke R, Qizilbash N, Peto R, Collins R; Prospective Studies Collaboration. Age-specific relevance of usual blood pressure to vascular mortality: a meta-analysis of individual data for one million adults in 61 prospective studies. Lancet 2002;360:1903-1913.

2. Adler AI, Stratton IM, Neil HA, et al. Association of systolic blood pressure with macrovascular and microvascular complications of type 2 diabetes (UKPDS 36): prospective observational study. BMJ 2000;321:412-419.

3. SPRINT Research Group, Wright JT Jr, Williamson JD, et al. A randomized trial of intensive versus standard blood-pressure control. N Engl J Med 2015;373:2103-2116.

4. Hajjar I, Kotchen TA. Trends in prevalence, awareness, treatment, and control of hypertension in the United States, 1988-2000. JAMA 2003;290:199-206.

5. Epstein M. Resistant hypertension: prevalence and evolving concepts. J Clin Hypertens (Greenwich) 2007;9(1 Suppl 1):2-6.

6. Egan BM, Zhao Y, Axon RN, Brzezinski WA, Ferdinand KC. Uncontrolled and apparent treatment resistant hypertension in the United States, 1988 to 2008. Circulation 2011;124:1046-1058.

7. Judd E, Calhoun DA. Apparent and true resistant hypertension: definition, prevalence and outcomes. J Hum Hypertens 2014;28:463-468.

8. Bangalore S, Fayyad R, Laskey R, et al. Prevalence, predictors, and outcomes in treatment-resistant hypertension in patients with coronary disease. Am J Med 2014;127:71-81.

9. Chobanian AV, Bakris GL, Black HR, et al. Seventh report of the Joint National Committee on Prevention, Detection, Evaluation, and Treatment of High Blood Pressure. Hypertension 2003;42:1206-1252.

10. McAdam-Marx C, Ye X, Sung JC, Brixner DI, Kahler KH. Results of a retrospective, observational pilot study using electronic medical records to assess the prevalence and characteristics of patients with resistant hypertension in an ambulatory care setting. Clin Ther 2009;31:1116-1123.

11. Falaschetti E, Chaudhury M, Mindell J, Poulter N. Continued improvement in hypertension management in England: results from the health survey for England 2006.
Hypertension 2009;53:480-486.

12. Persell SD. Prevalence of resistant hypertension in the United States, 2003-2008. Hypertension 2011;57:1076-1080.

13. Sim JJ, Bhandari SK, Shi J, et al. Characteristics of resistant hypertension in a large, ethnically diverse hypertension population of an integrated health system. Mayo Clin Proc 2013;88:1099-107.

14. Lee KN, Na JO, Choi CU, et al. Prevalence and characteristics of resistant hypertension at primary clinics in Korea: a nationwide cross-sectional study. Clin Hypertens 2016;22:4.

15. Choi SW, Kim MK, Han SW, et al. Apparent treatment-resistant hypertension among elderly Korean hypertensives: an insight from the HIT registry. J Hum Hypertens 2014;28:201-205.

16. Choi SI, Kim SK, Park S, et al. Prevalence of resistant hypertension and associated factors for blood pressure control status with optimal medical therapy using Korean ambulatory blood pressure monitoring registry data. Clin Hypertens 2016;22:8.

17. Black HR, Elliott WJ, Neaton JD, et al. Baseline characteristics and early blood pressure control in the CONVINCE trial. Hypertension 2001;37:12-18.

18. Dahlof B, Devereux RB, Kjeldsen SE, et al. Cardiovascular morbidity and mortality in the Losartan Intervention for Endpoint reduction in hypertension study (LIFE): a randomised trial against atenolol. Lancet 2002;359:995-1003.

19. Lloyd-Jones DM, Evans JC, Larson MG, O’Donnell CJ, Roccella EJ, Levy D. Differential control of systolic and diastolic blood pressure: factors associated with lack of blood pressure control in the community. Hypertension 2000;36:594-599.

20. Cushman WC, Ford CE, Cutler JA, et al. Success and predictors of blood pressure control in diverse North American settings: the antihypertensive and lipid-lowering treatment to prevent heart attack trial (ALLHAT). J Clin Hypertens (Greenwich) 2002;4:393-404.

21. Calhoun DA, Jones D, Textor S, et al. Resistant hypertension: diagnosis, evaluation, and treatment: a scientific statement from the American Heart Association Professional Education Committee of the Council for High Blood Pressure Research. Hypertension 2008;51:1403-1419.

22. Williams B, MacDonald TM, Morant S, et al. Spironolactone versus placebo, bisoprolol, and doxazosin to determine the optimal treatment for drug-resistant hypertension (PATHWAY-2): a randomised, double-blind, 
crossover trial. Lancet 2015;386:2059-2068.

23. Bramlage P, Pittrow D, Wittchen HU, et al. Hypertension in overweight and obese primary care patients is highly prevalent and poorly controlled. Am J Hypertens 2004;17:904-910.

24. Caro JJ, Speckman JL, Salas M, Raggio G, Jackson JD. Effect of initial drug choice on persistence with antihypertensive therapy: the importance of actual practice data. CMAJ 1999;160:41-46.

25. Mazzaglia G, Mantovani LG, Sturkenboom MC, et al. Patterns of persistence with antihypertensive medications in newly diagnosed hypertensive patients in Italy: a retrospective cohort study in primary care. J Hypertens 2005;23:2093-2100.

26. Ho PM, Magid DJ, Shetterly SM, et al. Importance of therapy intensification and medication nonadherence for blood pressure control in patients with coronary disease. Arch Intern Med 2008;168:271-276.

27. Brown MA, Buddle ML, Martin A. Is resistant hyperten- sion really resistant? Am J Hypertens 2001;14:1263-1269.

28. Santos LP, Moraes RS, Vieira PJ, et al. Effects of aerobic exercise intensity on ambulatory blood pressure and vascular responses in resistant hypertension: a crossover trial. J Hypertens 2016;34:1317-1324.

29. Whelton SP, Chin A, Xin X, He J. Effect of aerobic exercise on blood pressure: a meta-analysis of randomized, controlled trials. Ann Intern Med 2002;136:493-503.

30. Whelton PK, Carey RM, Aronow WS, et al. 2017 ACC/AHA/ AAPA/ABC/ACPM/AGS/APhA/ASH/ASPC/NMA/PCNA guideline for the prevention, detection, evaluation, and management of high blood pressure in adults: a report of the American College of Cardiology/American Heart Association task force on clinical practice guidelines. Hypertension 2018;71:e13-e115.

31. Carey RM, Calhoun DA, Bakris GL, et al. Resistant hypertension: detection, evaluation, and management: a scientific statement from the American Heart Association. Hypertension 2018;72:e53-e9o. 